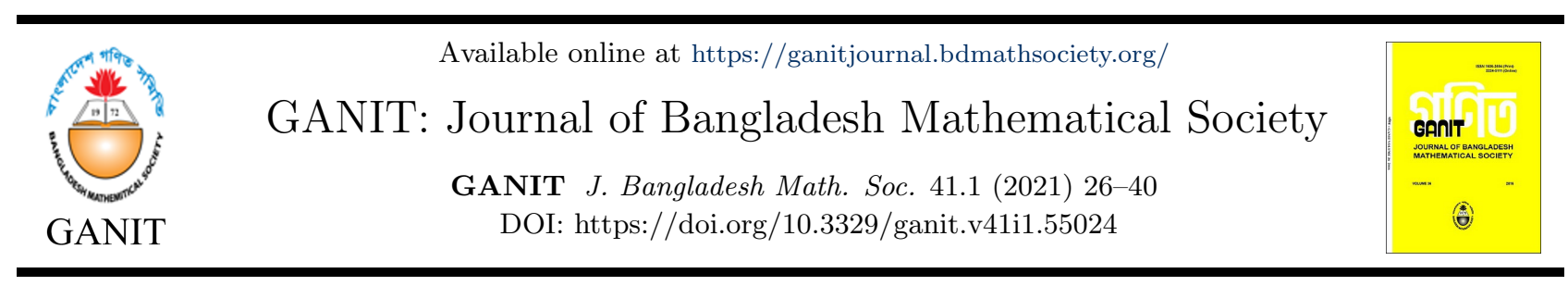

\title{
Pricing Exotic Options Using Some Lattice Procedures
}

\author{
Sadia Anjum Jumana *a and A B M Shahadat Hossain †b \\ ${ }^{a}$ Department of Applied Mathematics, University of Dhaka, Dhaka-1000, Bangladesh. \\ ${ }^{\mathrm{b}}$ Department of Applied Mathematics, University of Dhaka, Dhaka-1000, Bangladesh.
}

\begin{abstract}
In this work, we discuss some very simple and extremely efficient lattice models, namely, Binomial tree model (BTM) and Trinomial tree model (TTM) for valuing some types of exotic barrier options in details. For both these models, we consider the concept of random walks in the simulation of the path which is followed by the underlying stock price. Our main objective is to estimate the value of barrier options by using BTM and TTM for different time steps and compare these with the exact values obtained by the benchmark Black-Scholes model (BSM). Moreover, we analyze the convergence of these lattice models for these exotic options. All the results have been shown numerically as well as graphically.
\end{abstract}

(C) 2021 Published by Bangladesh Mathematical Society

Received: November 26, 2020 Accepted: March 18, 2021 Published Online: August 04, 2021

Keywords: Binomial tree model (BTM); Trinomial tree model (TTM); Black-Scholes Model(BSM); Exotic options; Barrier Option.

AMS Subject Classifications 2020:

\section{Introduction}

In the last few decades, derivatives such as options became quite important part in the financial market. In otherwords, pricing options has become one of the vital phenomenons in finantial mathematics. Note that, trading options begun in the Chicago Board Options Exchange (CBOE) in 1973 and after that numerous other exchanges started trading options all around the world [1]. In 1973, Fischer Black and Myron Scholes established the first thoroughly acceptable model for pricing option [2]. After that Robert C. Merton expannded their version in various significant ways in the same year [3].

The binomial lattice model to approximate option premium came out of a discussion between W.F. Sharpe and M. Rubinstein at a conference. Their proposed model consists of the principle that, if an economy with three securities can only obtain two future states, one such security will be redundant. This inspection engendered to a two-state model. This was the birth of the binomial tree model (BTM) introduced first by J.C. Cox, S.A. Ross and M. Rubinstein in 1979 [5]. Then it became a very popular and useful for pricing options due to its simplicity.

On the other hand, Trinomial tree model (TTM) was developed by Boyle [4] and then it was modified by

*Sadia Anjum Jumana. E-mail address: sadiajumana@gmail.com

${ }^{\dagger}$ A B M Shahadat Hossain. E-mail address: abmsh@du.ac.bd 
Kamrad and Ritchen [14]. In TTM there exist three variation states for the underlying asset price at a specific time interval namely, up, down, and remain same. This concept is comparatively more practical than the BTM and it provides not only more accurate results but also faster.

Exotic options are established to fulfil the particular needs of the investors who are using the instruments to control financial risk. Inspite of referring exotic options as a class of options, they are rather considered as options with more convoluted properties than ordinary put and call options. Barrier options are most likely to be the oldest of all exotic options and have been traded in the US market ever since 1967 [7].

Pricing barrier options using lattice procedures (BTM and TTM) can be quite tricky. Although using a large number of time steps can generate precise outcomes for the standard options, the similar outcome might not occur in valuing barrier options. The problem arises in locating the barrier with corresponding adjacent branches of nodes in the lattice. In case of the barrier locates between the branches of the lattice, significant amount of errors might occur. We have found few papers about pricing barriers in the literature; Peter H. Ritchken discussed trinomial procedure for pricing and hedging most types of exotic barriers [15, 16]. On the other hand, lattice procedures have been considered by many researchers to price various options as they are easy to understand and very efficient [12-14].

The paper is structured as follows. In Section 2, we give some definitions regarding the options that we have worked on this paper along with their payoff diagr ams. Then we present a significant relationship between call and put option known as Put-Call Parity. After that, we briefly discuss three diffrent option pricing formulas namely, the BSM, BTM and TTM in section 3. In section 4, we provide some important numerical as well as graphical results about barrier option pricing using these models. Finally, we conclude how BTM and TTM fit against real market data in comparison with the BSM for pricing barriers in section 5 .

\section{Preleminaries}

\subsection{Some Definitions}

Definition 1. (Option) Option can be defined as a contract between two parties which provides the buyer (owner) of the option the right (but not an obligation) to buy or sell the underlying asset for the settled cost (strike price) on or before expiring time (maturity time) of the contract [1].

There exist two fundamental types of options:

- Call option

- Put option

Definition 2. (Call Option) A Call Option provides the holder (buyer) the right (but not the liability) to purchase the underlying asset by a fixed date for a fixed price.

Definition 3. (Put Option) A Put Option provides the holder the right (but not the liability) to sell the underlying asset by a fixed date for a fixed price.

Definition 4. (European Option) The type of option that provides the holder (buyer) of the option the right to implement the option only at the expiry date, is called European Option.

Definition 5. (American Option) An American option, in comparison with the European option, can be implementd at any time before the expiry date.

\subsection{Payoff Diagrams}

In the following table we will discuss the payoff of the call (Long position) and the put (Long position) option $[1]$. 
Table 2.1: Payoff of European call and put Options

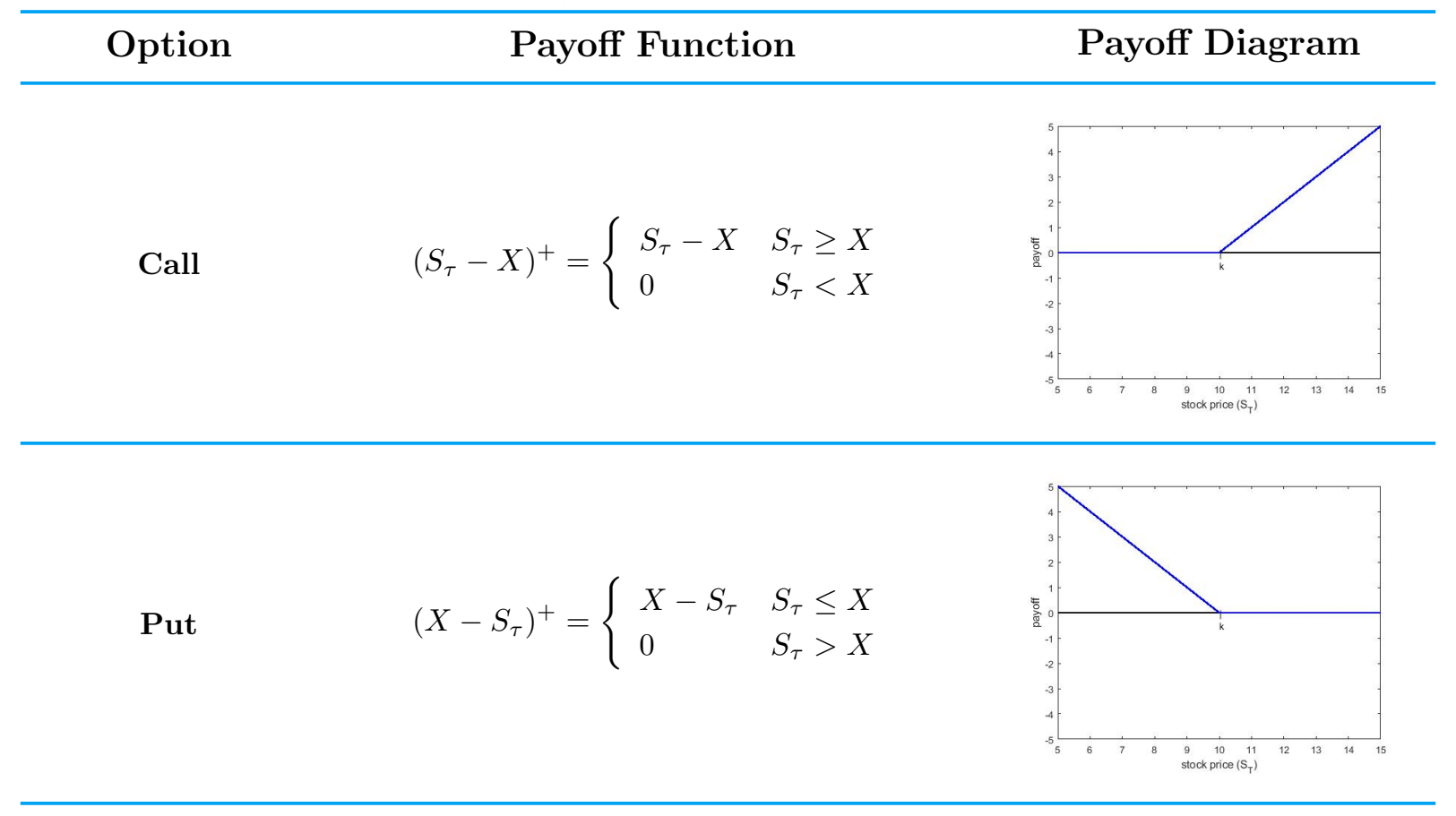

\subsection{Barrier Options}

Barrier options are regarded as one of the most fundamental kinds of path dependent exotic options. The exercise of the option depends on the underlying asset price crossing a definite barrier. Since the payoff is dependent on the path travelled by the asset price, it is called path dependent. However, the dependencey of the path is considered to be weak, since the only feature accounted is whether the barrier $B$ has been activated or not [6]. These options are attractive as they are comperatively less costly than that of the corresponding standard options.

Definition 6. (Barrier Option) The option for which the payoff depends on whether the price of the underlying asset hits a specific level during a specific time period, is called Barrier option [1].

\subsection{Types of Barrier Options}

A barrier can be hit from above or from below. Depending on this characteristic a barrier option can be classified into two types [7].

1. Knock-out Barrier Option: This type of Barrier option begins it's life as a typical call or put option, but it becomes invalid if the spot price ever hits a specific predetermined knock-out barrier, even before the expiry date.

Furthermore, depending on the position of the barrier with respect to the initial value of the underlying asset, the Knock-out barrier option can be classified in to two options.

- Down-and-out: The option which comes to an end when the underlying asset price diminishes to a predetermined level.

- Up-and-out: The option which comes to an end when the underlying asset price rises to a predetermined level.

2. Knock-in Barrier Option: This type of Barrier option begins its life inactive, and only becomes active when the underlying stock price hits the knock-in barrier, then it is regarded as a typical call or put option.

The Knock-in barrier option can also be classified in to two options. 
- Down-and-in: The option which becomes active when the underlying asset price decreases to a predetermined level.

- Up-and-in: The option which becomes active when the underlying asset price rises to a predetermined level.

In this paper we will only work with the Down-and-Out Barrier Call Option and Down-and-Out Barrier Put Option ,whose payoff functions and graphs are given below [8],

- Down-and-Out Barrier Call

$$
\left(S_{\tau}-X\right)^{+} \mathbb{1}_{\left\{\min _{0 \leq t \leq \tau} S_{t}>B\right\}}=\left\{\begin{array}{lll}
S_{\tau}-X & \text { if } & \min _{0 \leq t \leq \tau} S_{t}>X \\
0 & \text { if } & \min _{0 \leq t \leq \tau} S_{t} \leq X
\end{array}\right.
$$

Remark 7. Here, $\mathbb{1}$ is the indicator function of a set (which set, is ususally specified as an index under the 1) - a function that is equal to 1 if the argument belongs to the set, and 0 if it doesn't.

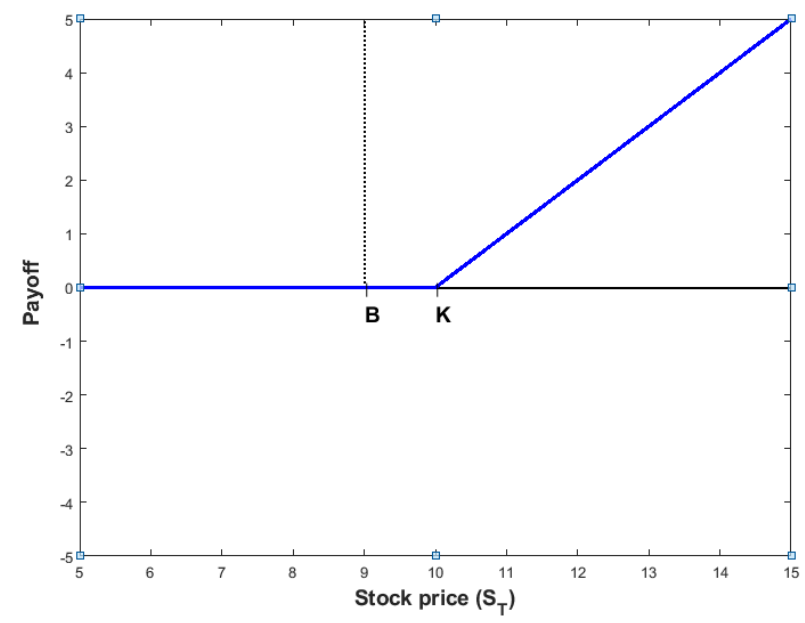

Figure 2.1: Payoff diagram of Down-and-Out Barrier Call Option

\section{- Down-and-Out Barrier Put}

$$
\left(X-S_{\tau}\right)^{+} \mathbb{1}_{\left\{\min _{0 \leq t \leq \tau} S_{t}>B\right\}}=\left\{\begin{array}{lll}
X-S_{\tau} & \text { if } & \min _{0 \leq t \leq \tau} S_{t}>X \\
0 & \text { if } & \min _{0 \leq t \leq \tau} S_{t} \leq X
\end{array}\right.
$$




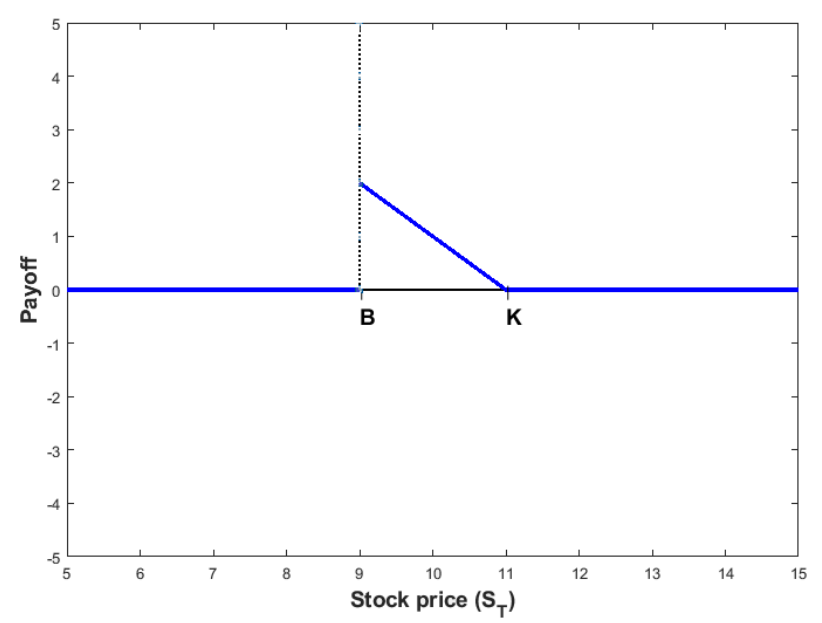

Figure 2.2: Payoff diagram of Down-and-Out Barrier Put Option

\subsection{Put-Call Parity}

The principle which explains the relation between the value of European call option $\left(C_{t}\right)$ and European put option $\left(P_{t}\right)$ with the same underlying asset $\left(S_{t}\right)$, expiry date $(\tau)$, and strike price $(X)$, is known as put-call parity [1]. Mathematically it is given by

$$
P_{t}+S_{t}=C_{t}+X e^{-r(\tau-t)}
$$

This relation states that the simultaneous holding of a long European call along with a short European put of the same class will provide the identical result as holding one forward contract on the same underlying asset, with the same expiry, and a forward price same as the strike price of the option. In case of the prices of the call and put options conflict such that this relationship refrain holding, there exists an arbitrage opportunity. i.e. sophisticated traders get the opprtunity to receive a risk-free profit [9].

\subsection{In-out Parity}

In-out parity can be interpreted as the representation of the put-call parity for barrier option. The main concept here is almost identical as in put-call parity (2.1). It states that, if the value of the "in" option is added with the value of the "out" option, then it results in the value of the European option (for both call and put)[17].

$$
\begin{aligned}
& C=C_{\text {in }}+C_{\text {out }} \\
& P=P_{\text {in }}+P_{\text {out }}
\end{aligned}
$$

\section{$3 \quad$ Methodology}

In this section, we will discuss the BSM, BTM and TTM option pricing models briefly that overviews the needs of this works. For details, readers are reffered [1].

\subsection{Notations}

Now we present some notations that are utilized in this paper as follows: 


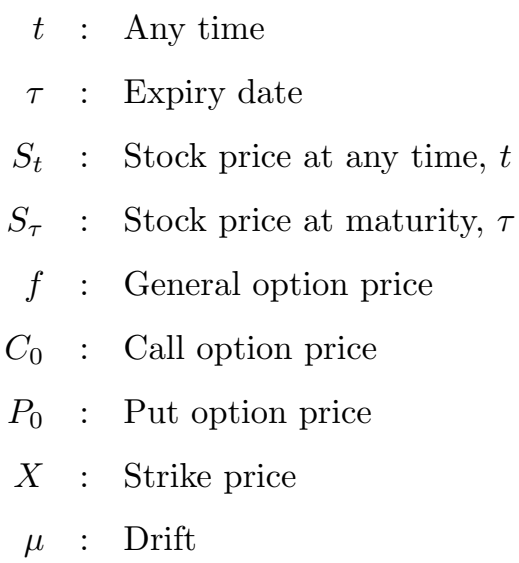

$$
\begin{aligned}
& r: \text { Risk-free interest rate } \\
& \sigma: \text { Volatility } \\
& u: \text { Up movement } \\
& d: \text { Down movement } \\
& p_{u}: \text { Probability to move up } \\
& p_{d}: \text { Probability to move down } \\
& N \quad: \text { Number of steps } \\
& B \quad: \text { Barrier of the stock price }
\end{aligned}
$$

\subsection{The Black-Scholes Model}

This model was presented by Fisher Black, Myron Sholes and Merton at the beginning of the 1970s for pricing European stock options. Since then it's been used as the standard model for pricing European options.[1]

Proposition 8. Let, $S$ be the asset price, which follows GBM process

$$
\frac{d S_{t}}{S_{t}}=\mu d t+\sigma d W_{t} \quad\left(W_{t} \text { is Wiener process }\right)
$$

and $f$ be the value of the option, that follows the following PDE

$$
\frac{\partial f}{\partial t}+\frac{1}{2} \sigma^{2} S^{2} \frac{\partial^{2} f}{\partial S^{2}}+r S \frac{\partial f}{\partial S}-r f=0
$$

Then the value of the call option is given by

$$
C(S, t)=S \Phi\left(\delta_{1}\right)-X e^{-r(\tau-t)} \Phi\left(\delta_{2}\right)
$$

where

$$
\begin{aligned}
& \delta_{1}=\frac{\ln (S / X)+\left(r+\frac{1}{2} \sigma^{2}\right)(\tau-t)}{\sigma \sqrt{(\tau-t)}} \\
& \delta_{2}=\frac{\ln (S / X)+\left(r-\frac{1}{2} \sigma^{2}\right)(\tau-t)}{\sigma \sqrt{(\tau-t)}}=\delta_{1}-\sigma \sqrt{(\tau-t)}
\end{aligned}
$$

The parameter $\Phi($.$) is called the cumulative probability distribution function corresponding to any standard$ normal random variable $z$,

$$
\Phi(z)=\frac{1}{\sqrt{2 \pi}} \int_{-\infty}^{z} e^{-\frac{1}{2} x^{2}} d x
$$

Remark 9. After obtaining the formula for valuing a European call option, we can apply the put-call parity (2.1) for establishing the value $P(S, t)$ of a European put option which is

$$
P(S, t)=X e^{-r(\tau-t)} \Phi\left(-\delta_{2}\right)-S \Phi\left(-\delta_{1}\right)
$$

Remark 10. The first accepted scientific formula to price a Down-and-Out Barrier Call option was suggested by Merton [3] and shortly after Reiner and Rubinstein came up with the formulas for pricing all four kinds of Barrier call and put options [10]. From their inspection it was found that, the value of a barrier option depends on the parameters of the benchmark Black-Scholes model, along with the barrier level, B. According to them the value of a Down-and-In Barrier Call option is stated as below

$$
C_{\text {down-in }}(S, t)=\left(\frac{S}{B}\right)^{1-\frac{2 r}{\sigma^{2}}}-C\left(\frac{B^{2}}{S}, \tau-t\right)
$$

where, $C\left(\frac{B^{2}}{S}, \tau-t\right)$ is determined making use of the equation (3.3). 
Having obtained that, the value of a Down-and-Out Call option can easily be calculated by utilizing the in-out parity (2.2), for $B<X, B \leq S$ and $0 \leq t \leq \tau$.

$$
\begin{aligned}
C(S, t) & =C_{\text {down-in }}(S, t)+C_{\text {down-out }}(S, t) \\
C_{\text {down-out }}(S, t) & =C(s, t)-C_{\text {down-in }}(S, t) \\
& =C(s, t)-\left(\frac{S}{B}\right)^{1-\frac{2 r}{\sigma^{2}}}-C\left(\frac{B^{2}}{S}, \tau-t\right)
\end{aligned}
$$

According to [11] the values of th barrier option can be obtained by using the formulas mentioned below.

\section{Down-and-Out Call:}

1. $X>B$ :

$$
C_{\text {down-out }}(S, t)=S\left(\Phi\left(\delta_{1}\right)-\beta\left(1-\Phi\left(\delta_{8}\right)\right)\right)-X e^{-r(\tau-t)}\left(\Phi\left(\delta_{2}\right)-\alpha\left(1-\Phi\left(\delta_{7}\right)\right)\right) .
$$

2. $X<B$ :

$$
C_{\text {down-out }}(S, t)=S\left(\Phi\left(\delta_{3}\right)-\beta\left(1-\Phi\left(\delta_{6}\right)\right)\right)-X e^{-r(\tau-t)}\left(\Phi\left(\delta_{4}\right)-\alpha\left(1-\Phi\left(\delta_{5}\right)\right)\right) .
$$

\section{Down-and-Out Put:}

1. $X>B$ :

$$
P_{\text {down-out }}(S, t)=-S\left(\Phi\left(\delta_{3}\right)-\Phi\left(\delta_{1}\right)-\beta\left(\Phi\left(\delta_{8}\right)-\Phi\left(\delta_{6}\right)\right)\right)+X e^{-r(\tau-t)}\left(\Phi\left(\delta_{4}\right)-\Phi\left(\delta_{2}\right)-\alpha\left(\Phi\left(\delta_{7}-\Phi\left(\delta_{5}\right)\right)\right)\right) .
$$

2. $X<B$ :

$$
P_{\text {down-out }}(S, t)=0
$$

$$
\begin{aligned}
& \text { where, } \alpha=\left(\frac{B}{S}\right)^{-1+\frac{2 r}{\sigma^{2}}}, \quad \beta=\left(\frac{B}{S}\right)^{1+\frac{2 r}{\sigma^{2}}} \text {. } \\
& \delta_{3}=\frac{\ln (S / B)+\left(r+\frac{1}{2} \sigma^{2}\right)(\tau-t)}{\sigma \sqrt{(\tau-t)}}, \quad \delta_{4}=\frac{\ln (S / B)+\left(r-\frac{1}{2} \sigma^{2}\right)(\tau-t)}{\sigma \sqrt{(\tau-t)}} \\
& \delta_{5}=\frac{\ln (S / B)-\left(r-\frac{1}{2} \sigma^{2}\right)(\tau-t)}{\sigma \sqrt{(\tau-t)}}, \quad \delta_{6}=\frac{\ln (S / B)-\left(r+\frac{1}{2} \sigma^{2}\right)(\tau-t)}{\sigma \sqrt{(\tau-t)}} \\
& \delta_{7}=\frac{\ln \left(S X / B^{2}\right)-\left(r-\frac{1}{2} \sigma^{2}\right)(\tau-t)}{\sigma \sqrt{(\tau-t)}}, \quad \delta_{8}=\frac{\ln \left(S X / B^{2}\right)-\left(r+\frac{1}{2} \sigma^{2}\right)(\tau-t)}{\sigma \sqrt{(\tau-t)}}
\end{aligned}
$$

\subsection{Binomial Tree Model}

A simple but convenient and quite popular procedure for valuing option requires constructing a binomial tree. It is basically a diagram based discrete time model demonstrating various possible paths accompanied by the asset prices throughout the life span of an option. It is considered that, the price of the stock follows a random walk. At every time step, there is a specific probability to move up by a definite percentage amount and a specific probability to move down by a definite percentage amount. As the number of steps of the tree increases, the time step gets smaller, and this model tends to the BSM [12].

Proposition 11. Let, $S_{n}^{m}$ and $f_{n}^{m}$ be the $n$-th possible values of stock price and option price at time-step $m \Delta t$ respectively which have the following representations

$$
S_{n}^{m}=u^{n} d^{m-n} S_{0}^{0} \quad n=0,1,2, \ldots, m,
$$

and,

$$
f_{n}^{m}=e^{-r \Delta t}\left[p f_{n+1}^{m+1}+(1-p) f_{n}^{m+1}\right] \quad n=0,1,2, \ldots, m
$$


Then the current option price $f_{0}$ is given by

$$
f_{0}=e^{-r \tau} \mathbb{E}_{p}\left[f_{\tau}\right]=e^{-n r \Delta t} \sum_{h=0}^{n} \frac{n !}{h !(n-h) !} p^{h}(1-p)^{(n-h)} f_{u^{h} d^{(n-h)}}
$$

where, $p=\frac{e^{r \Delta t}-d}{u-d}, u=e^{\sigma \sqrt{\Delta t}}, d=\frac{1}{u}, \quad u>1$.

Remark 12. The prices of european call and put option can be easily calculated by (3.15) with the following final conditions:

$$
f_{n}^{N}= \begin{cases}\max \left\{S_{n}^{N}-X, 0\right\} & \text { for Call } \\ \max \left\{X-S_{n}^{N}, 0\right\} & \text { for Put }\end{cases}
$$

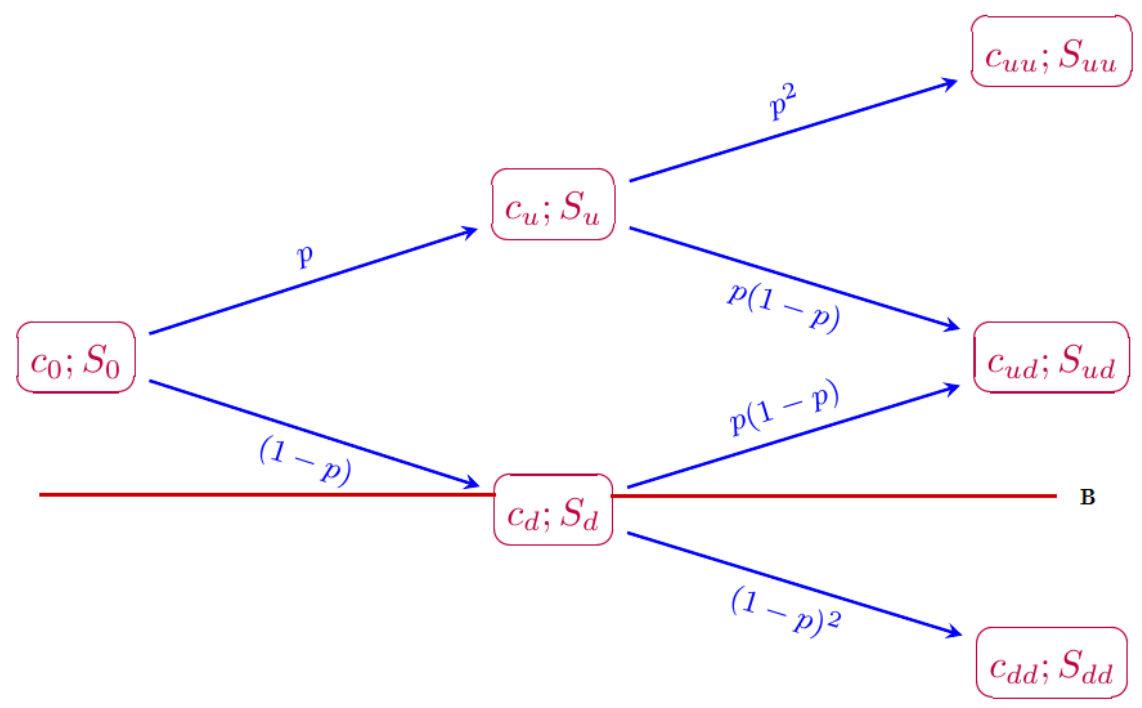

Figure 3.1: Two step Binomial tree of a Down-and-out Barrier Call option

Figure 3.1 represents a two step binomial tree for both the option price $c$ and stock price $S$ of a Down-and-out Barrier Call option. At each time step the prices may either go up by a factor $u>1$ accompanied by probability $p$ else go down by $d<1$ accompanied by probability $(p-1)$. The option terminates when the underlying stock price falls to a predetermined level, $B$.

\subsection{Trinomial Tree Model}

The BTM has turned out to be considerably out of date and is of little practical use. Therefore, a more advanced model known as the TTM came into account. This model also provides discrete representation of stock price movement, analogous to BTM. This ameliorates the BTM by enabling the price of stock to shift up, down or remain same with specific probabilities [13].

Proposition 13. Let, $S_{n}^{k}$ and $f_{n}^{k}$ be the $n$-th possible values of stock price and option price at time-step $k \Delta t$ respectively which have the following representations

$$
S_{n}^{k}=u^{h} d^{l} m^{n-h-l} S_{0}^{0} \quad n=0,1,2, \ldots, k,
$$

and,

$$
f_{n}^{k}=e^{-r \Delta t}\left[p_{u} f_{n+1}^{k+1}+p_{m} f_{n}^{k+1}+p_{d} f_{n-1}^{k+1}\right] \quad n=0,1,2, \ldots, k,
$$


Then the current option price $f_{0}$ is given by

$$
f_{0}=e^{-n r \Delta t} \sum_{h=0}^{n} \sum_{l=0}^{n-h} \frac{n !}{h ! l !(n-h-l) !} p_{u}^{h} p_{d}^{l} p_{m}^{(n-h-l)} f_{u^{h} d^{l} m^{n-h-l}}
$$

where, $u=e^{\lambda \sigma \sqrt{\Delta t}}, d=\frac{1}{u}, m=1, u>1$ and

$$
\left\{\begin{array}{l}
p_{u}=\frac{1}{2 \lambda^{2}}+\frac{r-\frac{1}{2} \sigma^{2}}{2 \lambda \sigma} \sqrt{\Delta t} \\
p_{d}=\frac{1}{2 \lambda^{2}}-\frac{r-\frac{1}{2} \sigma^{2}}{2 \lambda \sigma} \sqrt{\Delta t} \\
p_{m}=1-\frac{1}{\lambda^{2}}
\end{array}\right.
$$

are the risk neutral probabilities [14] [15].

Note that $\lambda<1$ implies $p_{m}<0$, which explains the condition $\lambda \geq 1$. In the case $\lambda=1$, we have $p_{m}=0$ and hence the trinomial tree reduces to a simple binomial tree. The Final conditions are given by Remark 12.

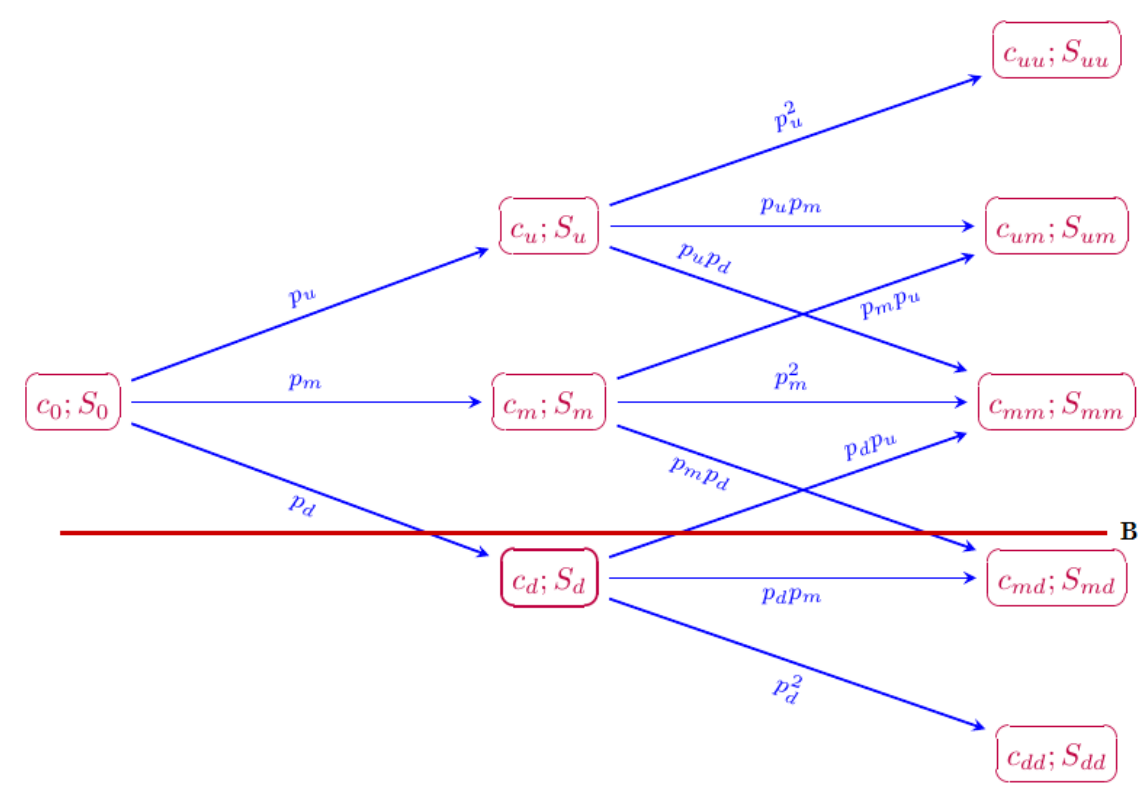

Figure 3.2: Two step trinomial tree of a Down-and-out Barrier Call option

Figure 3.2 represents a two step trinomial tree for both option price $c$ and stock price $S$ of a Down-and-out Barrier Call option. At each time step the prices changes by either a factor of $u>1$ with probability $p_{u}$ or $m$ with probability $p_{m}$ or $d<1$ with probability $p_{d}$. The option terminates when the underlying stock price decreases to a predetermined level, $B$.

\section{Results and Discussion}

In this section, we approximate Down-and-Out Barrier Call and Put option prices by BTM, TTM and BSM with the following data set [16]

$$
\left\{S_{0}=\$ 95, B=\$ 90, X=\$ 100, \sigma=0.25, r=0.10, \tau=1 \mathrm{yr}\right\} .
$$

Table 4.1 and Table 4.2 show the values of the Down-and-Out Barrier Call and Put options using CRR BTM and Kamrad-Ritchken TTM for increasing number of steps. 
Table 4.1: Barrier Call Option valuation using BTM and TTM

\begin{tabular}{cccccc}
\hline $\mathbf{N}$ & $\begin{array}{c}\text { BSM } \\
\text { (Call) }\end{array}$ & $\begin{array}{c}\text { BTM } \\
(\text { Call })\end{array}$ & $\begin{array}{c}\text { TTM } \\
(\text { Call })\end{array}$ & $\begin{array}{c}\text { Absolute } \\
\text { Error(BTM) }\end{array}$ & $\begin{array}{c}\text { Absolute } \\
\text { Error(TTM) }\end{array}$ \\
\hline 25 & & 8.8406 & 6.0069 & 2.8438 & 0.0101 \\
50 & & 7.2372 & 5.9942 & 1.2404 & $2.6 \times 10^{-3}$ \\
75 & & 6.2981 & 5.9899 & 0.3013 & $6.9 \times 10^{-3}$ \\
100 & & 7.5028 & 5.9997 & 1.506 & $2.9 \times 10^{-3}$ \\
125 & & 6.9839 & 5.9985 & 0.9871 & $1.7 \times 10^{-3}$ \\
150 & & 6.5601 & 5.9976 & 0.5633 & $8 \times 10^{-4}$ \\
175 & 5.9968 & 6.2087 & 5.9970 & 0.2119 & $2 \times 10^{-4}$ \\
200 & & 7.2299 & 5.9986 & 1.2331 & $1.8 \times 10^{-3}$ \\
250 & & 6.7074 & 5.9980 & 0.7106 & $1.2 \times 10^{-3}$ \\
300 & & 6.2903 & 5.9976 & 0.2935 & $8 \times 10^{-4}$ \\
350 & & 6.9599 & 5.9979 & 0.9631 & $1.1 \times 10^{-3}$ \\
400 & & 6.6501 & 5.9977 & 0.6533 & $9 \times 10^{-4}$ \\
450 & & 6.3819 & 5.9975 & 0.3851 & $7 \times 10^{-4}$ \\
500 & & 6.1456 & 5.9974 & 0.1488 & $6 \times 10^{-4}$ \\
\hline
\end{tabular}

Table 4.2: Barrier Put Option valuation using BTM and TTM

\begin{tabular}{cccccc}
\hline $\mathbf{N}$ & $\begin{array}{c}\text { BSM } \\
\text { (Put) }\end{array}$ & $\begin{array}{c}\text { BTM } \\
\text { (Put) }\end{array}$ & $\begin{array}{c}\text { TTM } \\
\text { (Put) }\end{array}$ & $\begin{array}{c}\text { Absolute } \\
\text { Error(BTM) }\end{array}$ & $\begin{array}{c}\text { Absolute } \\
\text { Error(TTM) }\end{array}$ \\
\hline 25 & & 0.1827 & 0.0322 & 0.1393 & 0.0112 \\
50 & & 0.0711 & 0.0334 & 0.0277 & 0.01 \\
75 & & 0.0570 & 0.0338 & 0.0136 & $9.6 \times 10^{-3}$ \\
100 & & 0.0948 & 0.0409 & 0.0514 & $2.5 \times 10^{-3}$ \\
125 & & 0.0703 & 0.0411 & 0.0269 & $2.3 \times 10^{-3}$ \\
150 & & 0.0612 & 0.0412 & 0.0178 & $2.2 \times 10^{-3}$ \\
175 & 0.0434 & 0.0446 & 0.0412 & $1.2 \times 10^{-3}$ & $2.2 \times 10^{-3}$ \\
200 & & 0.0828 & 0.0424 & 0.0394 & $1 \times 10^{-3}$ \\
250 & & 0.0623 & 0.0425 & 0.0189 & $9 \times 10^{-4}$ \\
300 & & 0.0491 & 0.0425 & $5.7 \times 10^{-3}$ & $9 \times 10^{-4}$ \\
350 & & 0.0729 & 0.0429 & 0.0295 & $5 \times 10^{-4}$ \\
400 & & 0.0615 & 0.0429 & 0.0181 & $5 \times 10^{-4}$ \\
450 & & 0.0529 & 0.0430 & 0.0105 & $4 \times 10^{-4}$ \\
500 & & 0.0477 & 0.0430 & $4.3 \times 10^{-3}$ & $4 \times 10^{-4}$ \\
\hline
\end{tabular}

It is clear from Table 4.1 that, call option valued by BTM oscillates towards the exact value obtained by BSM whereas, call option price obtained by TTM approximates the exact value correct upto two decimal places in only 50-th steps and as the step number rises, option value approaches to the exact value more accurately. Similar behaviour has been observed for barrier down and out put option in Table 4.2.

These behaviours are shown graphically in Figure 4.1- Figure 4.4. 


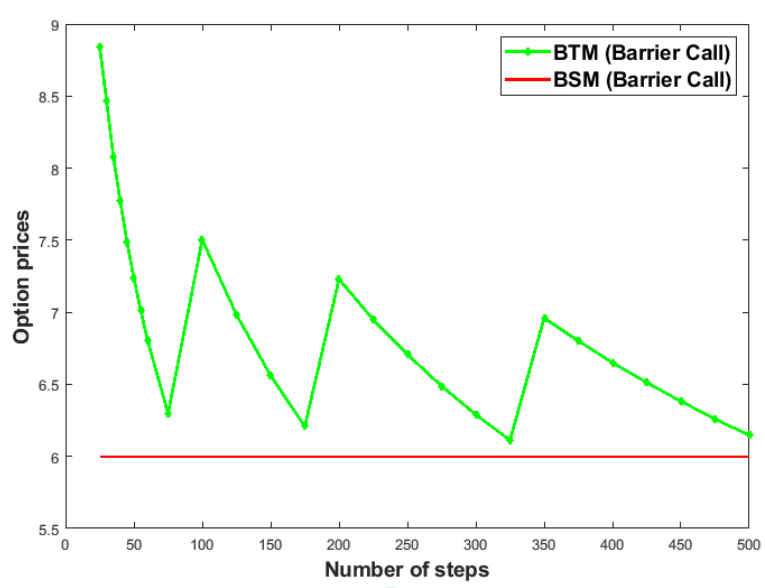

(a) Using BTM

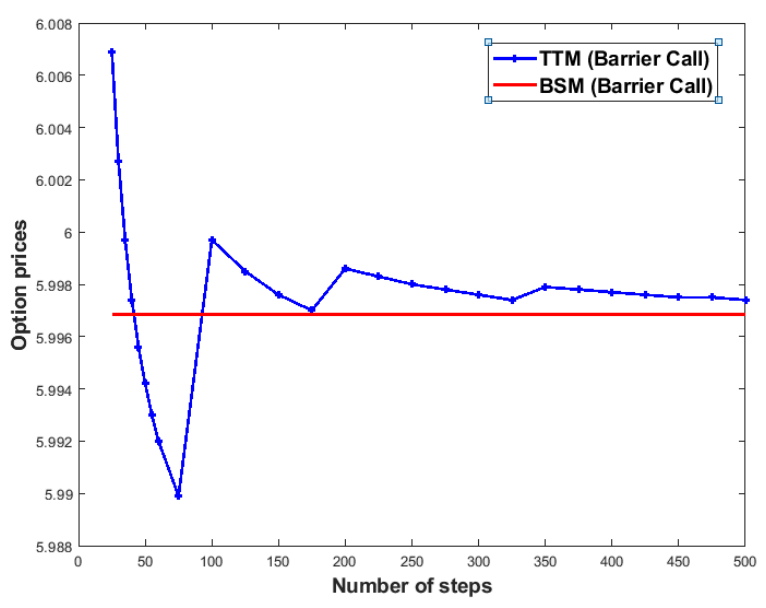

(b) Using TTM

Figure 4.1: Convergences for Barrier Call option prices obtained by BTM and TTM

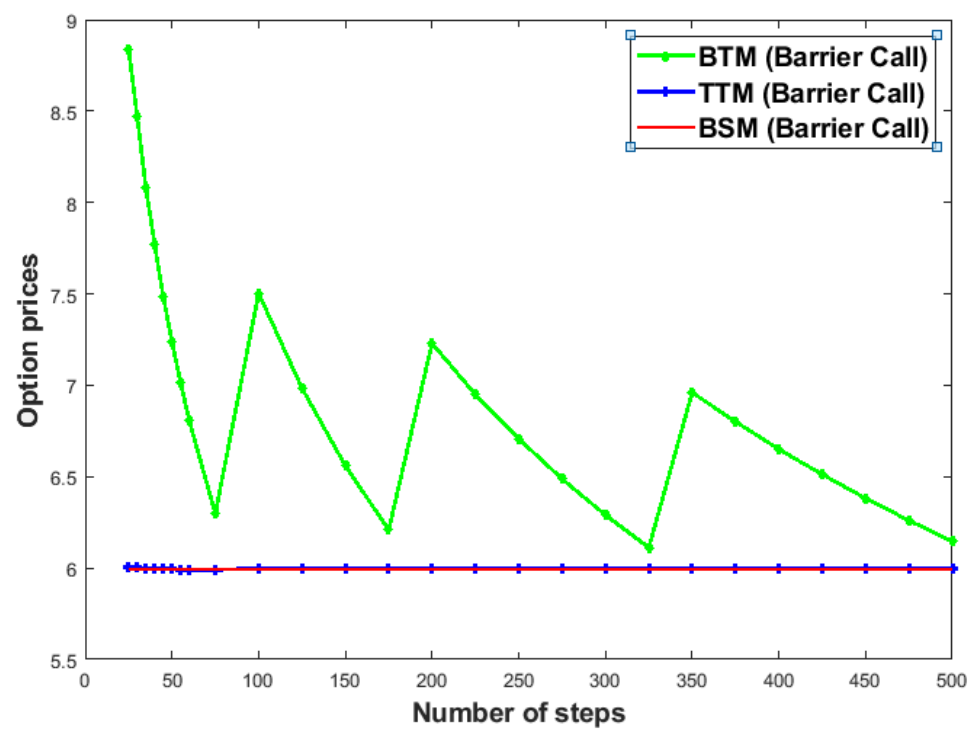

Figure 4.2: Comparison among Barrier Call option prices using different methods

From Figure 4.1 we observe that for a Down-and-Out Barrier Call option the values obtained by BTM oscillates towards the exact value obtained by BSM, whereas the values obtained by TTM initially oscillates and then converges towards the exact value.

While Figure 4.2 represents the comparison of the BTM and TTM values for Down-and-Out Barrier Call option. Clearly, it shows that the values obtained by TTM converges to the exact value more accurately than the values obtained by BTM. 


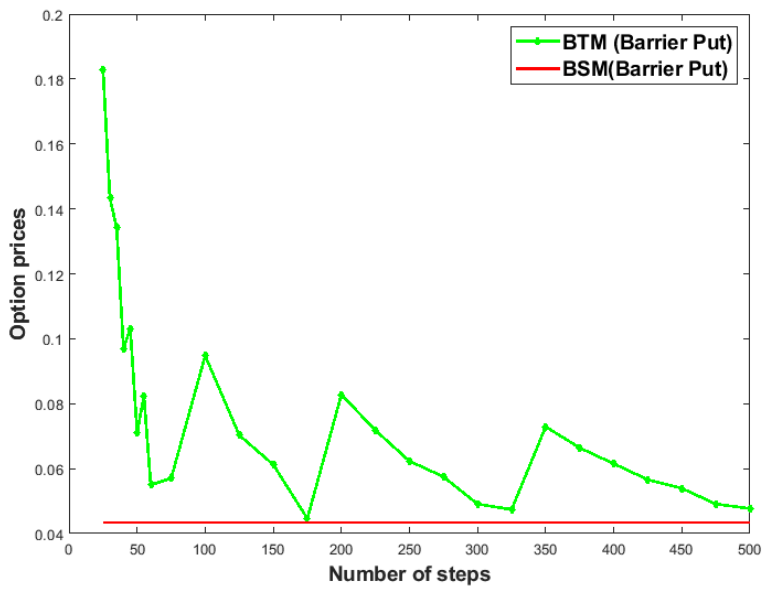

(a) Using BTM

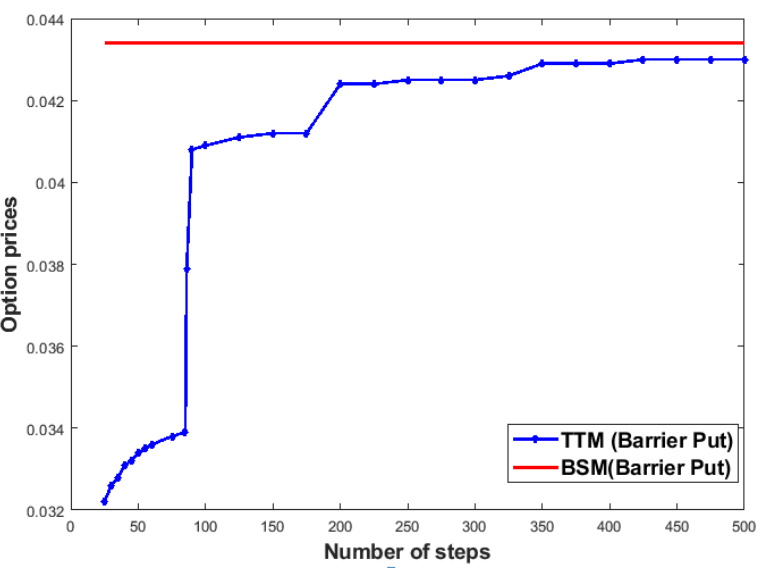

(b) Using TTM

Figure 4.3: Convergences for Barrier Put option prices obtained by BTM and TTM

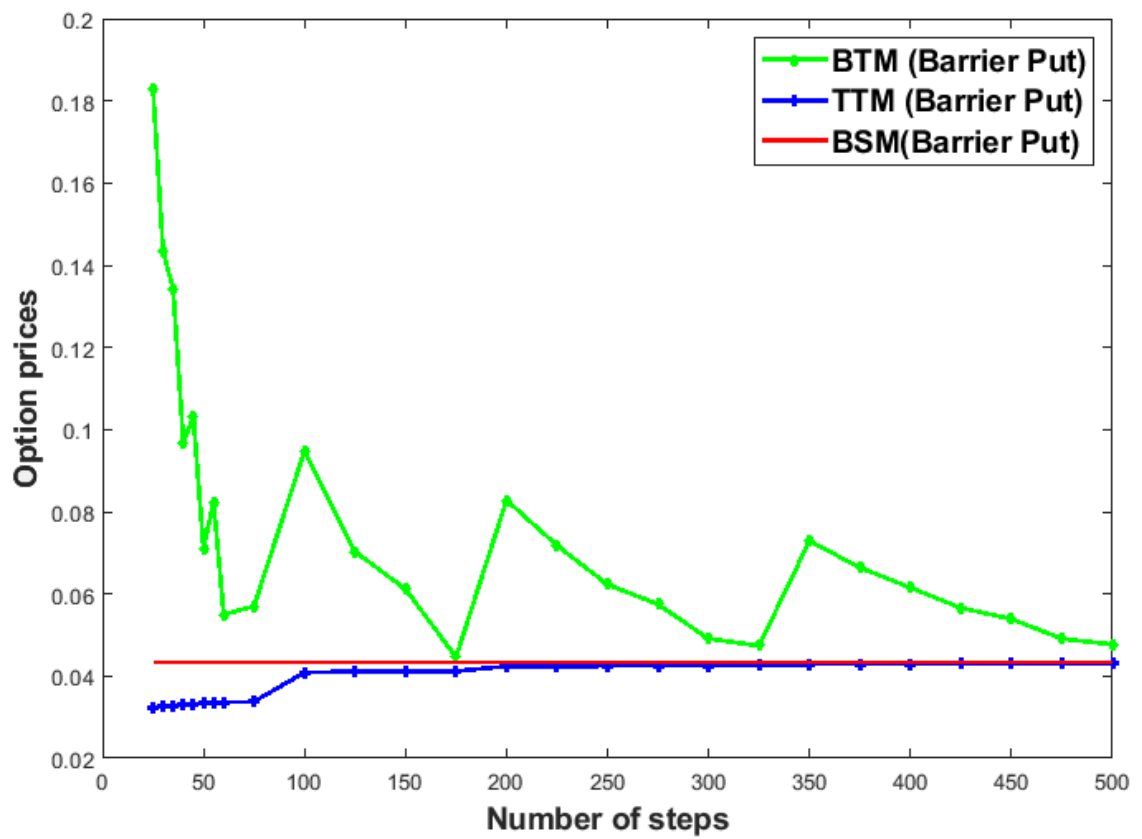

Figure 4.4: Comparison among Barrier Put option prices using different methods

From Figure 4.3 we observe that for a Down-and-Out Barrier Put option the the values obtained by BTM oscillates towards the exact value obtained by BSM, whereas the values obtained by TTM initially oscillates and then converges towards the exact value.

While Figure 4.4 represents the comparison of the BTM and TTM values for Down-and-Out Barrier Put option. Clearly, it shows that the values obtained by TTM converges to the exact value more accurately than the values obtained by BTM. 


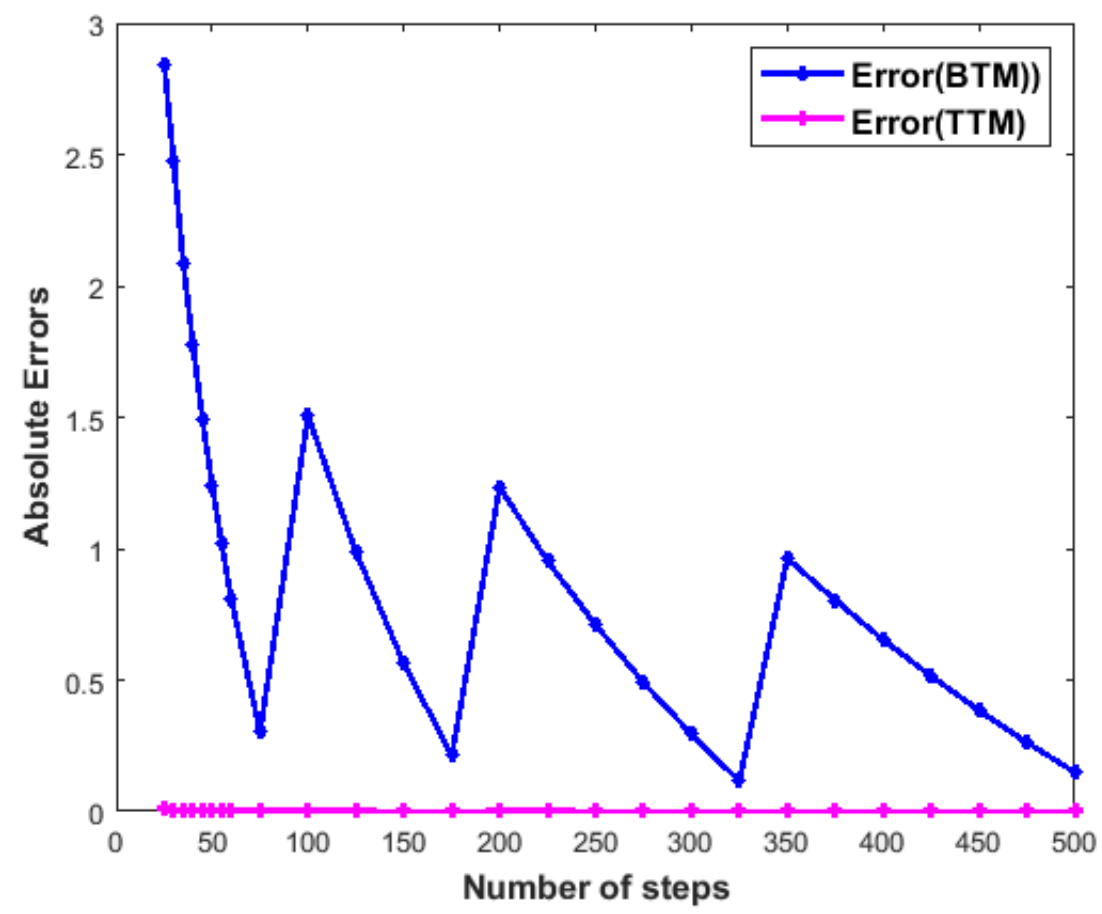

Figure 4.5: Comparison of Errors for different Barrier Call option prices

In Figure 4.5, we have shown the comparison of absolute errors for Down-and-Out Barrier Call option prices obtained by the BTM and TTM . It confirms that the TTM gives more accurate value than the BTM since the absolute errors of TTM tends to zero while the absolute errors of BTM is oscillating.

It is evident that both the lattice procedures BTM and TTM give very significant results against the data set mentioned at the beginning of this section. Our numerical results coincide with the results presented in [16]. In addition, we have shown the convergence of our results obtained by BTM and TTM with the BSM graphically (see Figure 4.1-Figure 4.5).

\section{Conclusion}

In this paper, we have got the opportunity to work on an exotic option, namely, Barrier option pricing. In particular, we approximate the Barrier option premium numerically using some lattice models such as BTM and TTM. For both these models we have got very significant results compare to that of BSM. We also found that TTM gives more accurate results than the BTM. Moreover, we can say that lattice models like BTM and TTM are satisfactorily flexible to price exotic options compare to other models such as Finite difference model, Monte carlo etc. We have generated some MATLAB coding to produce all our numerical and graphical results shown in this work (see Appendix). And finally, we can say that this study could be a useful guide line to evaluate other option price like Asian, Binary, Lookback etc.

\section{References}

[1] J. C. Hull., Options, Futures, and Other Derivatives. 8th ed.,Pearson Education Limited, 2012.

[2] F.Black and M.S.Scholes, The Pricing of Options and Corporate Liabilities, Journal of Political Economy, 81(3):637-654, May-June 1973.

[3] R.C.Merton, Theory of Rational Option Pricing, Bell Journal of Economics and Manage-ment Science, 4(1):141-183, Spring 1973.

[4] P. Boyle, Option valuation using a three jump process, Inter-national Options Journal,vol.3,pp.7-12,1986. 
[5] Merima Nurkanovic, The Split tree for option pricing, May 2017.

[6] P. Wilmott, J. Dewynne, and S. Howison. Option pricing: mathematical models and computation, Oxford: Financial Press, 1995.

[7] Chriss, N, Black-Scholes and Beyond Option Pricing Models, McGraw-Hill, NewYork, 1997.

[8] Nicolas Privault, Stochastic Finance An Introduction with Market Examples, Chapman and Hall/CRC Financial Mathematics Series, 2014.

[9] Hans R. Stoll, The Relationship Between Put and Call Option Prices, The Journal of Finance, 1969.

[10] Reiner, E. and Rubinstein, M, Breaking down the barriers, Risk 4(8): 28-35, 1991.

[11] Dewynne, J., Howison, S. and Wilmott, P., The mathematics of financial derivatives: A student introduction, Press Syndicate of the University of Cambridge, Cambridge,UK,1995.

[12] Cox, J., Ross, S. and Rubinstein, M., Option pricing: A simplified approach, Journal of financial economics September, 1979.(1): 1-35.

[13] Paul Clifford, Yan Wang, Oleg Zaboronski, Kevin Zhang, Pricing Options Using Trinomial Trees, University of Warwick, 2008.

[14] Ritchken, P. and B. Kamrad, Multinomial Approximating Models for Options with k State Variables, Management Science, Volume 37, Issue 12, 1640-1652, 1991.

[15] Ritchken, P., On Pricing Barrier Options, The Journal of Derivatives, Vol. 3, No. 2, 19-28, 1995.

[16] Gonçalo Nuno Henriques Mendes, Valuation of Barrier Options Through Trinomial Trees, 2011.

[17] Wilmott, P. Paul Wilmott introduces quantitative finance, 2nd ed, John Wiley \& Sons,London, UK.

\section{APPENDIX : Matlab Code for pricing Down and Out Barrier Option}

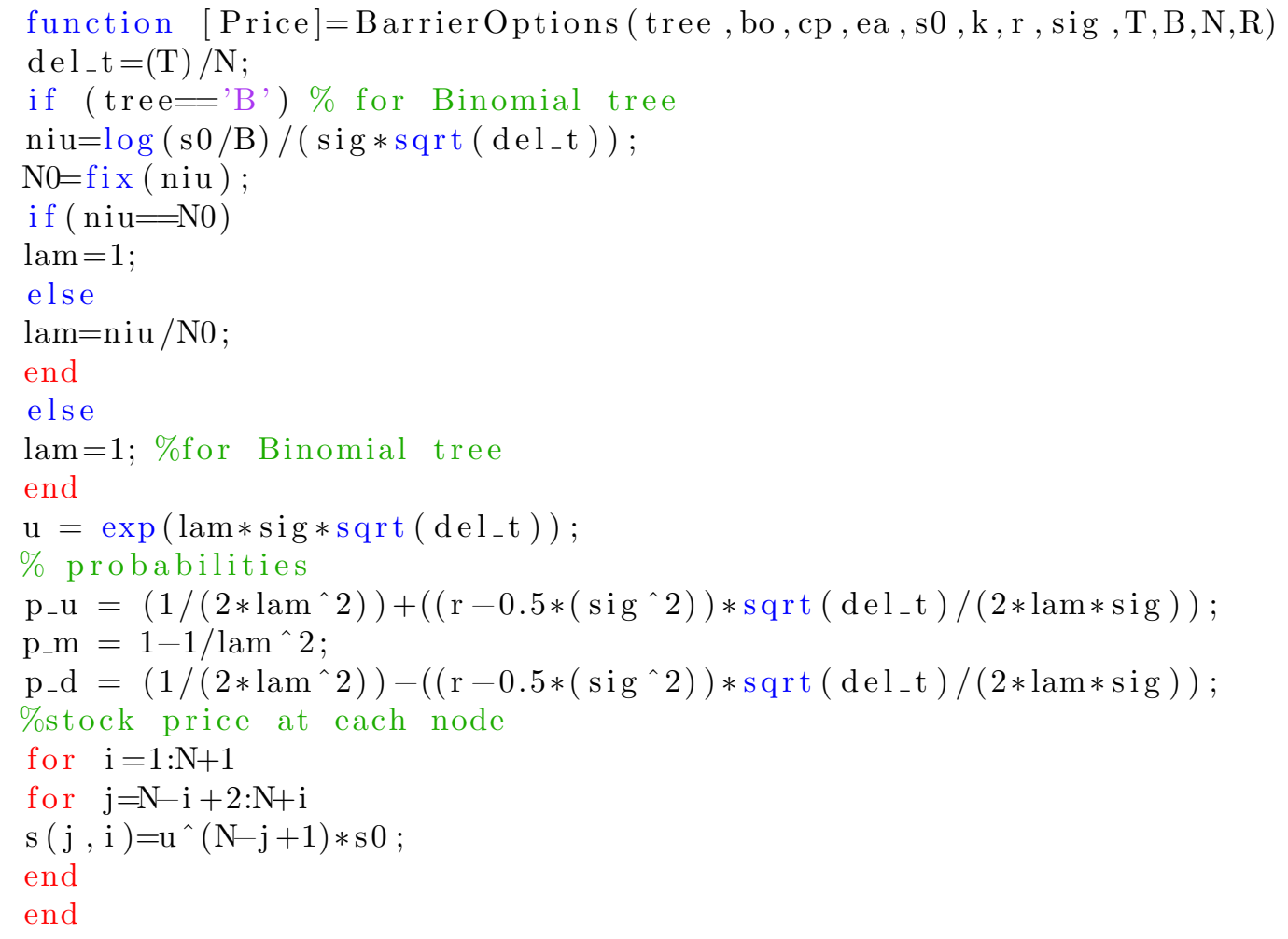




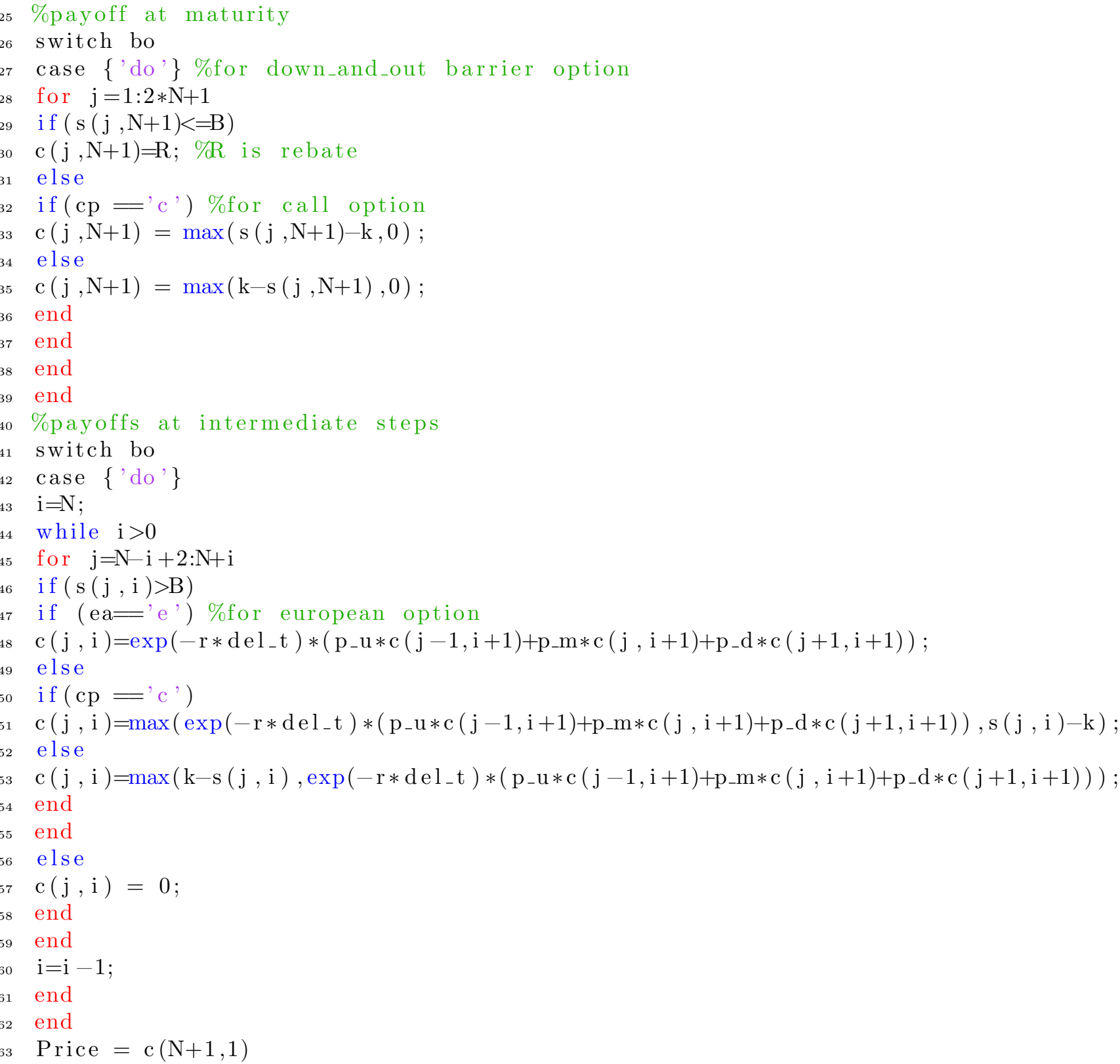

